\title{
High Precision Measurements Lend No Supporting Evidence of Previously Reported Large Verdet Constants for Olive Oil
}

\author{
William Brandon, Assoc. Prof. \\ Siva Mandjiny, Prof. \\ Killian McDonald \\ Sandra Huneycutt \\ Dakota Lee \\ University of North Carolina - Pembroke, USA
}

Doi: 10.19044/esj.2017.c1p11 URL:http://dx.doi.org/10.19044/esj.2017.c1p11

\begin{abstract}
Motivated by a previous report of surprisingly large Verdet constant measurements for olive oil at $633 \mathrm{~nm}$ and $650 \mathrm{~nm}$ (Shakir, et. al., 2013), and the practical utility of materials possessing such large values, we considered it worthwhile to validate those results. In this work, high precision Faraday rotation measurements were performed utilizing ac magnetic fields, phase sensitive detection, and a collection of diode lasers. Specifically, we measured the dispersion of the Verdet constant for a single brand of olive oil from 410 $\mathrm{nm}$ to $675 \mathrm{~nm}$. In addition, we determined the Verdet constant for eight different samples of olive oil at $654 \mathrm{~nm}$, very near the wavelength where the "anomalous" results, i.e. large Verdet constants, were reported. Our measurements of the Faraday rotations, and hence the determination of the respective Verdet constants, call into question those previously reported measurements. Generally, our results suggest that their experimental technique most likely led to inaccurate results for all five of the Verdet constant values they reported.
\end{abstract}

Keywords: Faraday rotation, Verdet constant, phase sensitive detection (PSD), magnetic rotatory dispersion, anomalous dispersion

\section{Introduction}

Materials with large Verdet constants are used in the fabrication of such devices as optical switches (Huang, et.al., 2015), rotators (Popescu, et.al., 2005), circulators (Dutton, 1998), isolators (Wunderlich, et.al., 1977 and Wilson 1991), modulators, laser gyroscopes (Merlo, et.al., 2000), satellite 
altitude monitors (Affoolderbach, et.al., 2002), and magnetic field/electric current sensors (Maystre, et.al, 1989). Most materials with large Verdet constants are expensive. There are inexpensive fluids with large Verdet constants, such as ferromagnetic fluids (Silva, et.al., 2012) or highly corrosive and/or toxic diamagnetic fluids (e.g. benzene and toluene) (Villaverde, et.al., 1979). Olive oil is both safe and inexpensive, therefore the surprising results (Shakir, et. al., 2013) are certainly worth validating. Essentially, they reported large values for the Verdet constant of olive oil at $633 \mathrm{~nm}$ and $650 \mathrm{~nm}$. Furthermore, based on the absorption spectra of olive oil near $670 \mathrm{~nm}$, one might naively suspect that such "anomalous" magnetic rotatory dispersion of the Verdet constant is quite plausible. However, our measurements do not confirm that claim. Additional considerations also lend credence to the results presented here and the subsequent conclusions.

Faraday rotation occurs when linearly polarized light traverses a transparent medium influenced by a magnetic field with directional components parallel, or anti-parallel, to the light propagation direction (Jenkins and White, 2001). Essentially, the electric field defining the plane of polarization exhibits a rotation (i.e. induced optical activity) due to the electronic response of the medium. The rotation of the polarized light is a magneto-optical phenomenon named after Michael Faraday (Barr, 1967) and is ultimately caused by a difference in propagation speeds between left and right circularly polarized photons, when one considers linearly polarized light as a linear combination of these two circular basis states. The parameter characterizing the rotation is the Verdet constant, $V$, expressed as $V=\square \square / B l$ (Jenkins and White, 2001), with $\square \square$ being the angle at which the polarized light is rotated, $B$ the magnetic field acting upon the sample of length, $l$. Verdet constants are material based and also depend on sample temperature and the wavelength of the light, and hence due to the wavelength dependence, are dispersive (i.e. magnetic rotatory dispersion). In this work we tested several different brands of olive oil and determined the Verdet constant at $654 \mathrm{~nm}$, which is very near the $650 \mathrm{~nm}$ wavelength where the largest value was reported (Shakir, et.al., 2013) for eight different olive oil samples. The Verdet constants for one of these samples were obtained at multiple wavelengths, and hence its dispersion characterized. Finally, our relatively straight forward Faraday rotation experiments include various aspects of optics and electromagnetism (Turvey, 1993, Briggs, et.al., 1993, Jain, et.al., 1999, Valev, et.al., 2008), and with the addition of an ac magnetic field and corresponding experimental methodology exploiting the phase sensitive lock-in method regarding data analysis of signals buried in noise (Jain, et.al., 1999, Valev, et.al., 2008), such investigations lend themselves quite well to training advanced undergraduates and graduate students in physics, engineering, chemistry and material science. 


\section{Apparatus}

The experimental setup (Fig. 1) allowed Faraday rotation measurements using a collection of laser diodes (Thorlabs CPS series: $450 \mathrm{~nm}$, $532 \mathrm{~nm}, 638 \mathrm{~nm}, 654 \mathrm{~nm}, 675 \mathrm{~nm}$ ). In addition, two laser pointers (eBay: 405 $\mathrm{nm}, 594 \mathrm{~nm}$ ) were also used with a regulated power supply (RSR HY3003) and a series current limiting resistor, which helps to minimize intensity fluctuations and drift. All laser wavelengths were measured (Photon Control SPM-002-X). In most cases we utilized laser powers of 1-5 $\mathrm{mW}$ (Thorlabs PM100D, S120VC). Appropriate lenses were employed for those lasers displaying rather large beam divergences. Next, an aperture to block unwanted back reflections, and a film polarizer to aid in manipulating the intensity, were placed just after the laser. Highly polarized light was obtained using a Nicole prism (length $\sim 3 \mathrm{~cm}$ ), scavenged from an old polarimeter. The air core solenoid (Pasco SE-7585) with inner diameter of $5.5 \mathrm{~cm}$ and a length of 14.5 $\mathrm{cm}$, allowed ample room to insert a custom made cuvette housing. A rectangular quartz cuvette providing $10 \mathrm{~mm}$ sample length (1 $\mathrm{mm}$ walls) was used for sample containment. A sinusoidal voltage output from the lock-in amplifier (Stanford Research Systems, SR-830), provided the internal reference frequency for the PSD, in addition to the solenoid current, which was amplified with a (QSC PLX-1202) power amplifier. The current was monitored by a benchtop multimeter (Vichy VC8145). Upon traversing the sample, the laser beam was directed through another aperture and baffle tube (not shown) to minimize stray light entering the detector. The analyzer (Thorlabs GL5-A, Glan-Laser Polarizer), was set at $45^{\circ}$ relative to the polarization direction to maximize the PSD signal $\left(V_{a c}\right)$. Finally, the resulting laser beam intensity was monitored with a photodetector (Thorlabs PDA100A). The transimpedance design of this detector provides near optimum impedance matching conditions when splitting the signal as voltage inputs (i.e. PSD signal, $V_{a c}$, and auxiliary signal, $V_{d c}$ ) to the SR-830 lock-in amplifier.

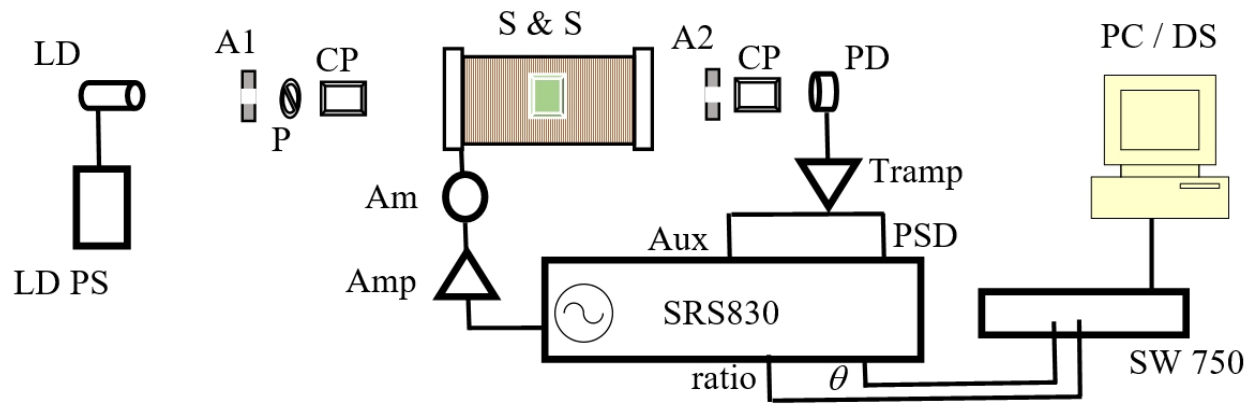

Figure 1. PSD Faraday Rotation Apparatus: LD-laser diode, LD PS-laser diode power supply, A1-aperture, P-film polarizer, CP-crystal polarizer (Nicole prism), S\&S-solenoid with sample, A2-aperture, CP-crystal polarizer (Glan-laser) polarizer, PD-photodetector, 
Tramp-transimpedance amplifer, Amp-current amplifier, Am-ammeter, SRS830-lock-in amplifier [inputs: Aux- $V_{d c}$ (avg. sig.), R- $V_{A C}$ (PSD sig.), outputs: ( ) - AC voltage \& ref freq, ratio $=\mathrm{R} / \mathrm{Aux}=V_{A C} / V_{d c}, \square$ - phase $]$, SW750-Science Workshop 750 interface, PC/DS, computer with Data Studio software.

The magnetic field was calibrated (Fig. 2) with a longitudinally configured hall sensor (Magnetic Instrumentation Inc., Model 907 Gaussmeter), in RMS mode. A jig was constructed allowing air core solenoids to be calibrated with up to $\mathrm{mm}$ step precision along the solenoid axis. Most of the data was acquired utlizing a current of about 3.5 amperes at a frequency of $85 \mathrm{~Hz}$, which is very near the geometric mean of potential noise sources at the fundamental $(60 \mathrm{~Hz})$ and second harmonic $(120 \mathrm{~Hz})$ frequencies associated with US mains. With these parameters (i.e. $I=3.5 \mathrm{~A}, f=85 \mathrm{~Hz}$ ) the magnetic field intensity of approximately 150 Gauss was nearly uniform across the sample (Fig 3). Finally, the front panel outputs of the lock-in amplifier were fed into a PC interface (Pasco Science Workshop 750) and software (Pasco Data Studio) was used to calculate the Verdet constants. Essentially, the apparatus is automated - aside from the solenoid current supplied as user input.

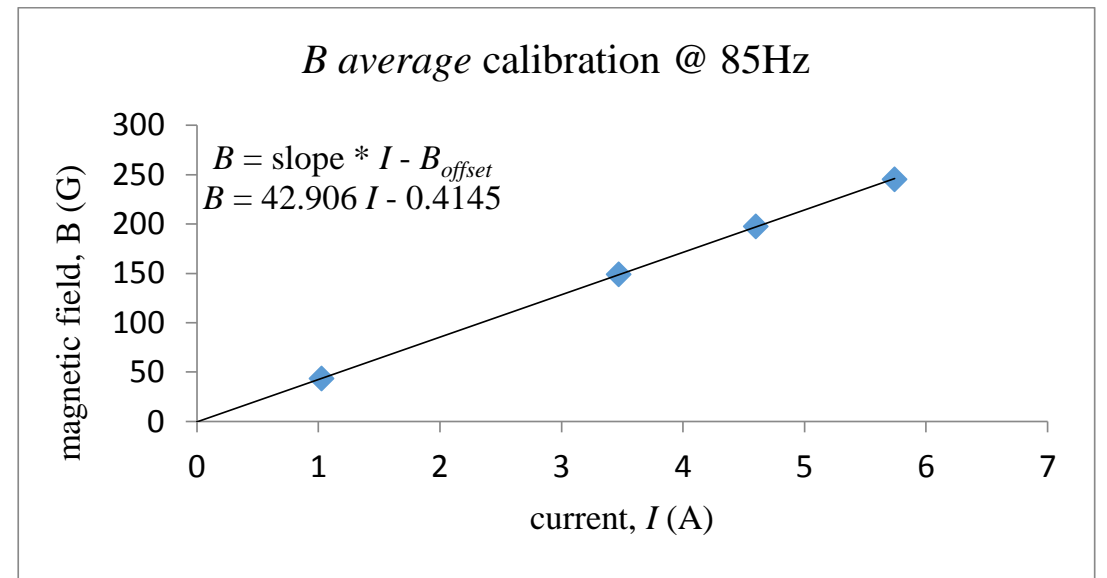

Figure 2. The average magnetic field strength, $B$ (RMS), measured across the $1.2 \mathrm{~cm}$ sample cuvette as a function of current, $I$. The variation of the magnetic field at these values of electric current was very small (see Fig. 3) 


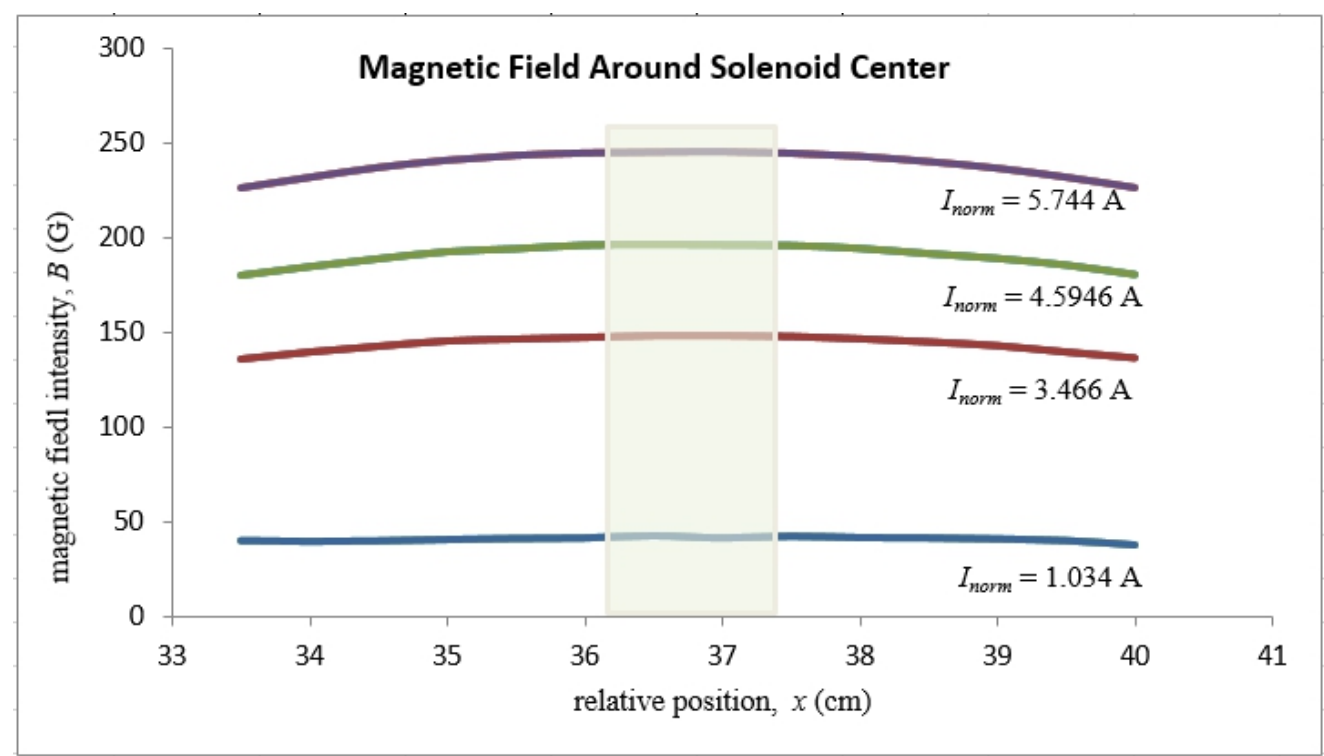

Figure 3. Magnetic field strength (RMS) vs. relative position for several currents. Note that the magnetic field strength across the cuvette and sample $(\sim 1.2 \mathrm{~cm}$ path length shown as a rectangle in the middle of the graph) is nearly uniform.

\section{Theory (Practical Aspects)}

Lock-in amplifiers (LIAs) record an input signal as RMS volts. It has been shown that the rotation, in radians, induced by a modulating magnetic field, follows from (Jain, et.al., 1999)

$\square \square=V_{a c} / 2 V_{d c}$,

where $V_{a c}$ is the voltage associated with the lock-in's PSD signal (R), and $V_{d c}$ corresponds to the average laser intensity (auxiliary input) (notation - Valev, et.al., 2008). In our apparatus these voltage signals result from the laser intensities being monitored with the photodiode and amplified via the transimpedance design of the PDA100A, before being fed into the front and back panel lock-in inputs using a BNC T-splitter. We should point out that we have seen no evidence of an "internal leak" from the internal reference frequency generator to the auxiliary inputs for the SRS 830 lock-in amplifier as claimed previously (Valev, et.al., 2008). We suspect that this deleterious behavior was actually due, in their experimental investigation, to a photodetector/amplifier impedance mismatch at low light intensities, which manifested as a correlation between the PSD signal (R) and the phase. We intend to fully address their claim in a future study.

It should be pointed out that an earlier paper describing a Faraday rotation measurement using polarity reversal of dc magnetic fields (Briggs, et.al., 1993) gives an expression that is indeed very similar to Equation 1. The experimental technique described in that paper is certainly superior to that 
used by Shakir, et.al. Acquiring measurements with both magnetic field directions (parallel and anti-parallel to the light propagation), in addition to a null current measurement, would clarify the unavoidable problems associated with fluctuations in laser output and photodetector drift. In contrast, Equation 1 , subsequently utilized and elaborated upon in a phase sensitive detection scheme (Jain, et. al., 1999) is extremely efficacious for Faraday rotations in that it was developed specifically for PSD and it corresponds to a direct measurement of the optical rotation while providing real time normalization to any fluctuations in the light intensity and, to a somewhat lesser extent, fluctuations in photodetector output. The expression is actually an approximation, yet it is completely valid and well within random errors for the small rotations, all less than 5 minutes ( $\sim 0.0015$ radians) of optical rotation, that occur in our apparatus (Fig. 1). Finally, the technique is suitable for signal to noise ratios approaching $10^{-5}$, which present themselves mainly as the ratio of the PSD signal, $V_{a c}(\mathrm{R})$ buried in the noise of the average signal, $V_{d c}$ (Aux), when the appropriate apparatus is employed (Fig. 1). Therefore, it is entirely possible to measure Faraday rotations approaching $10^{-5}$ radians utilizing the simple "Jain, et.al. prescription" if one employs appropriate devices - mainly a transimpedance amplified photodetector set with appropriate gain.

\section{Experimental Procedure}

After calibrating the solenoid (Figs. 2 and 3), the magnetic field strength is determined as a function of the monitored current supplied to the coil. A parameter, $\square$, is defined as the discrete sum of the magnetic field along the length of the sample, vis. $\square=\square \square B l$. The data is recorded and the Verdet constant follows from $V=\square \square s / \square$, where $\square_{s}$ is the Faraday rotation of the sample (i.e. olive oil). Of course, the contribution of the cuvette to the total Faraday rotation must also be taken into account. Essentially, the Faraday rotation of the empty cuvette, $\square_{c}$, must be subtracted from the Faraday rotation of the sample and the cuvette, $\square_{s+c}$, at every wavelength. That is, $\square_{s}=\square_{s+c}-$ $\square c$. The precision of the "Jain et.al. prescription" is further demonstrated by the reasonable values for the Verdet constants we obtained for the quartz cuvette (see Fig. 4). Those Verdet calculations for the walls of the cuvette resulted from Faraday rotations of less than one minute $(\sim 0.3 \mathrm{mrad})$. Finally, researchers present Verdet constants utilizing a variety of units. The most common of these are $\mathrm{rad} / \mathrm{T} . \mathrm{m}, \mathrm{deg} / \mathrm{T} . \mathrm{m}$, or min/G.cm, where $1 \mathrm{~T}=10^{4} \mathrm{G}$, and $1 \mathrm{rad}=[180 / \square] \mathrm{deg}=[180(60) / \square \square \min$.

\section{Results}

The Verdet constants for one of the brands of olive oil (Wild Oats) at the wavelengths produced by our collection of laser diodes, shown in Figure 4 and Table 1, follow a similar trend to that of typical materials, such as water 
(International Critical Tables, 1929) and quartz (i.e. cuvette walls). The higher values at the shorter wavelengths reflect normal magnetic rotatory dispersion in the same manner as the overwhelming majority of diamagnetic fluids (International Critical Tables, 1929), (Smithsonian Critical Tables, 1969) and Villaverde, et.al., 1979). There is no evidence of anomalous magnetic rotatory dispersion, and hence an increasing value of the Verdet constant, in the red region of the spectrum as claimed (Shakir, et.al., 2013).

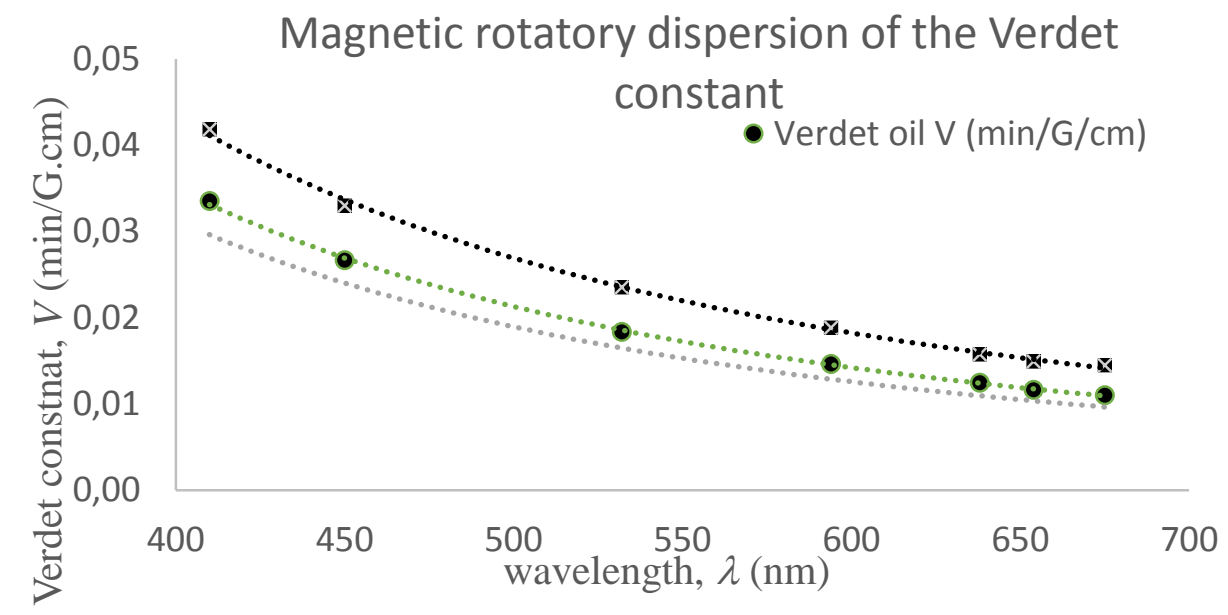

Figure 4. The Verdet constant of virgin olive oil (Wild Oats) vs. wavelength. The data obtained for the olive oil (center plot) shows normal magnetic rotatory dispersion. The Verdet constant for quartz (upper plot) is also shown since the Faraday rotation for the empty quartz cuvette had to be measured and then subtracted from the total rotation (sample + cuvette) according to $\square_{s}=\square_{s+c}-\square_{c}$. A trend line for water, combined data (International Critical Tables, 1929) and Smithsonian Critical Tables, 1969) - bottom plot is also presented in this visible range of wavelengths. Power law fits were arbitrarily assigned to each data set as a visual aid.

\begin{tabular}{|c|c|c|c|}
\hline $\begin{array}{c}\text { wavelength } \\
\square(\mathrm{nm})\end{array}$ & $\begin{array}{c}\text { Verdet constant } \\
V(\mathrm{~min} / \mathrm{G} / \mathrm{cm})\end{array}$ & $\begin{array}{c}\text { Verdet constant } \\
V(\mathrm{rad} / \mathrm{T} / \mathrm{m})\end{array}$ & $\begin{array}{c}\text { Verdet constant } \\
V(\mathrm{deg} / \mathrm{T} / \mathrm{m})\end{array}$ \\
\hline 410 & $0.0335(2)$ & $9.75(8)$ & $558(4)$ \\
450 & $0.0269(2)$ & $7.76(6)$ & $445(4)$ \\
532 & $0.01833(14)$ & $5.33(4)$ & $306(2)$ \\
594 & $0.01460(12)$ & $4.25(3)$ & $243.5(1.9)$ \\
638 & $0.01247(10)$ & $3.63(3)$ & $208.0(1.7)$ \\
654 & $0.01163(9)$ & $3.38(3)$ & $193.9(1.5)$ \\
675 & $0.01099(9)$ & $3.20(3)$ & $183.2(1.5)$ \\
\hline
\end{tabular}

Table 1. The table values for the Verdet constants of virgin olive oil (Wild Oats) displayed in the most common units. Random uncertainties accumulated from statistics for the 
rotation measurements, the magnetic field calibration, and specifications of the cuvette wall thickness (i.e. actual sample length). Due to the impressive precision afforded by this experimental technique, the contribution from the Faraday rotation measurement is the least of the three relative uncertainties $\square \square / \square \square \square B / B$, and $\square l / l$. The total propagated uncertainties were estimated at just under $1 \%$ for most of the final tabulated Verdet constants, as discussed in the section: Propagated Uncertainties.

Interestingly, the absorption spectrum of a few brands of the olive oils we tested (Fig. 5) utilizing an Ocean Optics spectrometer do show an increase near $670 \mathrm{~nm}$. This absorption peak appears to result from chlorophyll (inset Fig. 5). Based on the underlying connection between the refractive index and absorption characteristics for transparent materials one might expect this absorption peak to manifest itself as an increase in Faraday rotation (i.e. anomalous-like dispersion). Nevertheless, we see no evidence of such behavior.

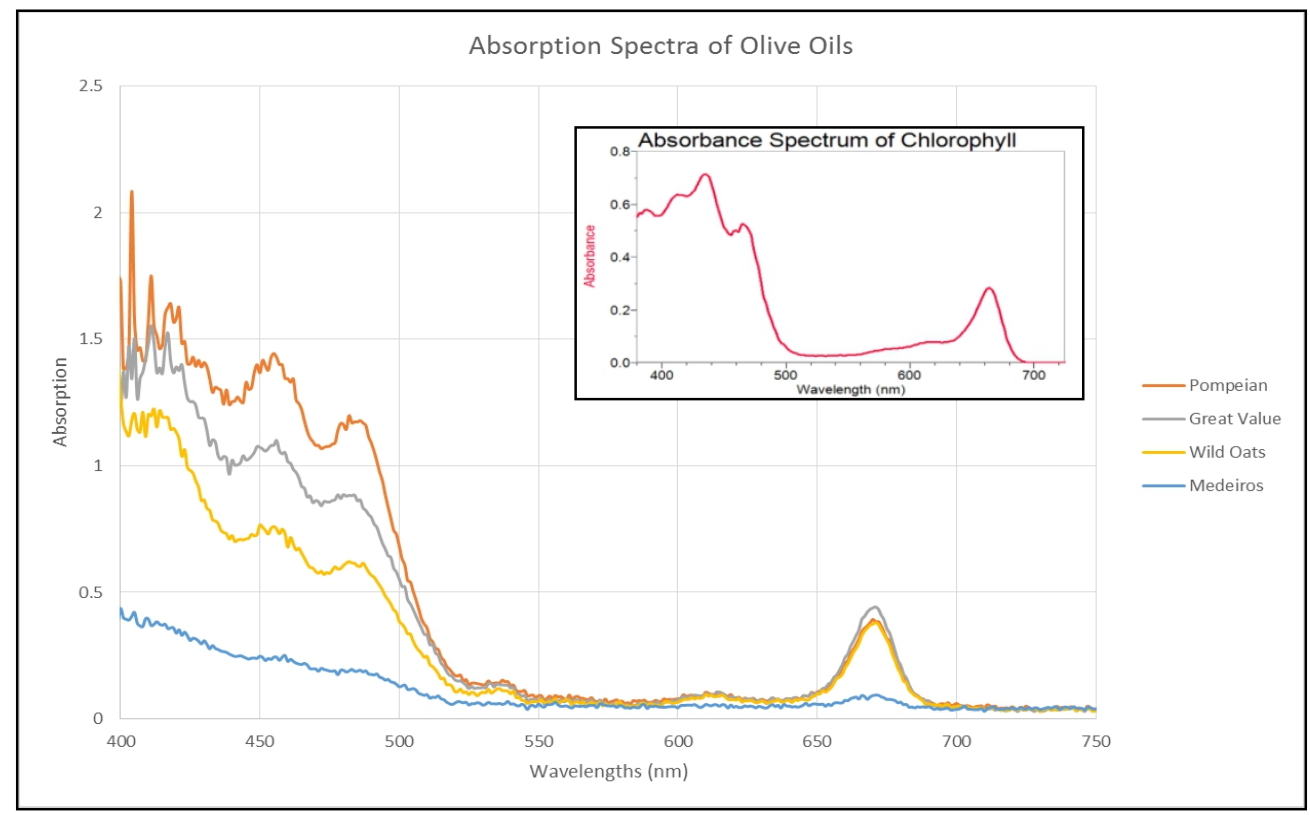

Figure 5. Absorbance spectrum for several different brands of the olive oils tested. The absorbance peaks located near $670 \mathrm{~nm}$ are due to chlorophyll (inset). Such behavior in the absorbance spectra of olive oil naively suggests a possible anomalous response in the dispersion of the Verdet constant. However, all eight of the olive oils tested (Table 2 below) show no increase in the corresponding magneto-optical response.

Based on Shakir, et.al.'s data, in particular the rather large Verdet constant value at $650 \mathrm{~nm}(\sim 99 \mathrm{rad} / \mathrm{T} . \mathrm{m}$ or $0.17 \mathrm{~min} / \mathrm{G} . \mathrm{cm})$, Verdet constants for a variety of olive oil samples near this wavelength were determined since one cannot expect all brands of olive oil to have the same ingredients. Utilizing the $654 \mathrm{~nm}$ laser diode, eight different brands (Table 2) were tested. All had similar values, with no large Verdet constants. The relatively small variations 
between the different brands would be expected on account of the different chemical composition of the various samples. However, all of our Verdet constants at this wavelength ranged from about thirteen to seventeen times smaller than that reported by Shakir, et.al.

\begin{tabular}{|c|c|}
\hline Brand & Verdet Constant \\
\hline Wild Oats & $0.0108(2)$ \\
Carlini & $0.00996(7)$ \\
Casa Di Oliva & $0.01181(12)$ \\
Food Lion & $0.0129(2)$ \\
Medeiros & $0.01232(13)$ \\
Great Value & $0.01214(14)$ \\
Pompeian & $0.01174(8)$ \\
Great Value & $0.01172(7)$ \\
\hline
\end{tabular}

Table 2. The Verdet constants of eight different brands of olive oil measured at $654 \mathrm{~nm}$. The small differences reflect the slight variation in ingredients associated with each respective brand.

These values are presented in $\mathrm{min} / \mathrm{G} . \mathrm{cm}$. Shakir, et.al. reported a value of $\sim 0.17$, which is about 13 to 17 times higher thanour values, utilizing these units.

It is highly unlikely that any non-linear optical effects (i.e. multiphoton interaction) would ensue for a $\mathrm{CW}$ laser power near $114 \mathrm{~mW}$, which coincides with that reported for the $650 \mathrm{~nm}$ laser (Shakir, et.al. 2013). Nevertheless, we tested one of our brands (Wild Oats) utilizing yet another laser diode at 652 $\mathrm{nm}$ (eBay) with a laser power of approximately $120 \mathrm{~mW}$. The Faraday rotation measurements, and hence the Verdet constant calculation, agreed with that obtained at low power $(\sim 0.01159 \mathrm{~min} / \mathrm{G} . \mathrm{cm})-$ essentially, no enhancement. In order to carry out this measurement, we applied three strips of Scotch Magic tape, acting as a light diffuser, just at the entrance of the photodetector baffle to mitigate against saturation, and a corresponding nonlinear detector response.

\section{Propagated Uncertainties}

The relative error due to uncertainty in the measurements follows from

$$
\square V / V=\left[(\square \square / \square)^{2}+(\square B / B)^{2}+(\square l / l)^{2}\right]^{1 / 2}
$$

where $\square \square$ was obtained from statistics (i.e. the standard deviation of five measurements, consisting of three minute runs acquired at every wavelength), 
and the corresponding relative uncertainty in rotation, $\square \square / \square$, (largest $\sim 0.2 \%$ ) was found to be significantly less than the relative uncertainties for the magnetic field $(0.8 \%)$, obtained from the calibration fit, and sample length $(0.5 \%)$, obtained from the cuvette specifications. Therefore, we expect uncertainties of just under $1 \%$ for nearly all measured values. Of course, any systematic errors resulting from the accuracy, and subsequent creep from manufacturer calibration of the lock-in, gauss meter, current meter, and the 750 interface, are not included in the previous expression. However, based on many self-consistency checks against other instruments within the specific parameters of our experiments, in addition to performing measurements with water, we found the responses of those instruments to be quite accurate and reproducible. In fact, one should certainly verify experimental accuracy with samples of water, since its Verdet constant is quite well-known (International Critical Tables, 1929).

\section{Conclusions}

Despite the absorption spectrum (Figure 5) and the earlier report of a large Verdet constant for olive oil in the red portion of the spectrum (Shakir, et. al., 2013)

1) the dispersion of the Verdet constant for the Wild Oats sample of olive oil shows no enhancement in the red spectral region of interest (Figure 3) and appears as "normal" magnetic rotatory dispersion, when compared to other common substances.

2) all samples tested at $\sim 650 \mathrm{~nm}$, which is near the wavelength where the significantly large value of the Verdet constant was reported, show no correspondingly large values (Table 3 ).

3) a variety of laser powers (CW mode) were tested on several brands to eliminate possible non-linear optical effects for the laser power reported, even if unlikely at such CW laser powers, and no anomalous responses were measured.

4) the absorption spectra shows a peak at $670 \mathrm{~nm}$, not $650 \mathrm{~nm}$. However, again, we found no enhanced Verdet constant at $675 \mathrm{~nm}$ for several brands of olive oil.

Finally, Table 3 suggests that most, if not all of the Shakir, et.al. data is highly suspect. None of their values are in agreement with those obtained utilizing our accurate methodology and high precision apparatus. Fitting our data (Figure 1) with a power law (the trend line in Fig. 1) allows us to compare our data at the specific wavelengths indicated, and measured by Shakir, et.al. Based on typical magnetic rotatory dispersion curves, one might expect this trend to extend quite accurately into the near infrared portion of the spectrum (i.e. near $805 \mathrm{~nm}$ ). 


\begin{tabular}{|c|c|c|}
\hline $\begin{array}{c}\text { wavelength } \\
(\mathrm{nm})\end{array}$ & $\begin{array}{c}\text { Shakir, } \text { et. al. } \\
\text { Verdet constant } \\
(\mathrm{rad} / \mathrm{T} . \mathrm{m})\end{array}$ & $\begin{array}{c}\text { This work } \\
\text { Verdet constant } \\
(\mathrm{rad} / \mathrm{T} . \mathrm{m})\end{array}$ \\
\hline 405 & 15.381 & 9.89 \\
532 & 0.1638 & 5.39 \\
632.8 & 33.3716 & 3.67 \\
650 & 98.6808 & 3.45 \\
804.3 & 1.0936 & 2.15 \\
\hline
\end{tabular}

Table 3. Comparison of our Verdet constants, numerically calculated from a power law fit (see Fig. 1), with those reported by Shakir, et.al. For additional reference, (Abu-Taha, et.al.,

2013) also reported Verdet constants of olive oil at $525 \mathrm{~nm}$ - "It is seen that the Verdet constant decreases appreciably from $227 \mathrm{deg} / \mathrm{T} . \mathrm{m}$ (3.96 rad/T.m) for less older sample to $165 \mathrm{deg} / \mathrm{T} . \mathrm{m}(2.88 \mathrm{rad} / \mathrm{T} . \mathrm{m})$ for older one."

While it is difficult, at best, to explain the suspect results of Shakir, et. $a l$., a few underlying effects that could cause errors include: etalon effects, saturation of polarizer, and electronic noise. However, any potential inaccuracies most likely result from fluctuations in laser power and photodetector response. Based on our reasonable values of the Verdet constants and Shakir et.al.'s reported magnetic field strengths $(550 \mathrm{G}$ maximum) and sample length $(1 \mathrm{~cm})$, one is able to estimate that they were attempting to measure anywhere from 5-20 minutes of angular rotation using a DC magnetic field with polarizers set at a relative angle of 45 degrees (for maximum change). In this particular type of experiment such a measurement is difficult, if not impossible, on account of a signal, which is essentially buried in noise. Utilizing their method, there is no way to normalize the signal corresponding to the angular change, and this is a major problem when the light intensity and the photodetector suffer from unavoidable fluctuations. They made no reference as to the reproducibility of these measurements, nor to the type of photodetector used. Also, the level of precision as implied by the number of significant digits reported is quite extreme. The reported precision of those values, considering the crudeness of the apparatus, is unacceptable - even in a rigorous intermediate-level teaching lab. Apparently, the journal reviewers missed this oversight. Finally, one might expect that it is highly unlikely that a "special" ingredient in their brand of olive oil would enhance the magneto-optical response, particularly when armed with the knowledge and experience that nearly all diamagnetic fluids possessing high Verdet constants are highly toxic and/or corrosive (e.g. toluene, benzene, carbon disulfide - Villaverde, et.al 1979).

The Verdet constant of olive oil, exhibits normal magnetic rotatory dispersion - similar to that of water, glass, quartz, etc. (i.e. nearly all optically 
transparent materials). There are only slight differences in the Verdet constants for the different brands of oil, as would be expected. Our results do not confirm the claim of Shakir et.al. In spite of not witnessing any enhancement for the Verdet constant of olive oil, our background research in this investigation did suggest that measurements involving the Verdet constants of

1) oleic, oleanic, and linoleic acid, and

2) chlorophyll (see inset in Figure 5 graph)

would comprise two distinct sets of experiments, both affording better control of chemical composition, and with a high potential of garnering interesting and useful information in the realm of magneto-optical chemistry.

\section{Acknowledgements}

The authors would like to thank the Research Initiative for Scientific Enhancement (funding under Grant \# 510215) and the Department of Chemistry and Physics, University of North Carolina at Pembroke (miscellaneous support: Carolyn Parsons and Hope Bullock).

\section{References:}

1. Shakir, Ali Adnan, Reiam D. AL- Mudhafa, Anwaar A. AL-Dergazly, Verdet constant measurement of olive oil for magnetic field sensor. Int. J. Adv. EEE 2 (3), pp. 362-368, 2013

2. Huang, Yi, Xuekun Bai, Zhaopu Chai, Fufei Pang, Jianxiang Wen, and Tingyun Wang, A magneto-optical fiber device based on fluid-filled photonic crystal fiber, Proceedings International Conference on Optical Instruments and Technology: Optical Sensors and Applications (Beijing, China), 2015

3. Popescu, Alexandru; Walther, Thomas. On the potential of Faraday anomalous dispersion optical filter as high-resolution edge filters. Laser Physics. 15 (1); pp. 55-60, 2005

4. Dutton, Harry J. R., Understanding Optical Communications (International Technical Support Organization), http://www.redbooks.ibm.com, 1998

5. J. A. Wunderlich and L. G. Deshazer, 'Visible Optical Isolator Using ZnSe,' Appl. Opt. 16, 6, pp. 1584-1587, 1977.

6. Wilson, D.K., Optical isolators adapt to communication needs," Laser Focus World 27 (4), pp. 175-180 (1991)

7. Merlo, Sabina, Michele Norgia, and Silvano Donati, Fibre gyroscope princples, in Handbook of Fibre Optic Sensing Technology (edited by José Miguel López-Higuera), John Wiley \& Sons Ltd, 2000

8. Affoolderbach, C., M. Stahler, S. Knappe, and R. Wyanads, Appl. Phys. B: Laser Opt. 2002, 75, pp. 605 
9. Maystre, F., and A. Bertholds, Magneto-optic current sensor using a helical-fiber Fabry-Perot resonator,” Opt. Lett. 14, pp. 587-589, 1989

10. Silva, R.M., H. Martins, I. Nascimento, J.M. Baptista, A.L. Ribeiro, J. L. Santos, P. Jorge,and O. Frazão, Optical Current Sensors for High Power Systems: A Review, Appl. Sci. 2(3), pp. 602-628, 2012

11. Villaverde, A.B., and D.A. Donatti, Verdet Constants of Liquids with Pulsed Magnetic Field. J. Chem. Phys. 71, pp. 4021-4024, 1979

12. Jenkins, F. A. and H. E. White, Fundamentals of Optics, Fourth Edition, McGraw-Hill Science/Engineering/Math, 2001.

13. Barr, E.S., Men and milestones in optics. V Michael Faraday, App. Opt. 6 p. 631,1967

14. Turvey, K., Determination of Verdet constant from combined ac and dc measurements. Rev. Sci. Inst. 64 6, pp. 1561-1568 1993.

15. Briggs, Peterson, Liquid cell Faraday modulator. Am. J. Physics, 61, pp.186-187, 1993

16. Jain, A., J. Kumar, F. Zhou, and L. Li, A simple experiment for determining Verdet constants using alternating current magnetic fields. Am. J. Physics, 67 (8), pp. 714-717, 1999

17. Valev, V.K., J. Wouters, and T. Verbiest, Precise measurements of Faraday rotation using ac magnetic fields. Am. J. Physics, 76, pp. 626629,2008

18. National Research Council of the United States of America, International Critical Tables of Numerical Data, Physics Chemistry and Technology, Vol. VI. McGraw-Hill, New York, 1st ed., p. 427, 1929.

19. W.E. Forsythe, Smithsonian Critical Tables, $9^{\text {th }}$ ed., Smithsonian Institution Press, Washington, D.C., 1969

20. Abu-Taha, M.I., M.A. Halasa, M.M. Abu-Samreh, On the Usage of the Faraday Effect as an Authentication Technique for Vegetable Oils. Journal of Modern Physics, 4, pp. 230-235, 2013 